\title{
Terapia adjunta con prednisona para pacientes con neumonía adquirida en la comunidad: un ensayo clínico aleatorizado, doble ciego, multicéntrico controlado con placebo
}

\author{
Joint therapy with prednisone for patients with \\ community-acquired pneumonia: a random, double- \\ blind, multicenter, placebo-controlled clinical trial
}

Abraham Alí Munive MD. (1); Alberto Zhong Lin, MD. ${ }^{(2)}$

(1)Médico internista, Neumólogo. Jefe Neumología Adultos. Fundación Neumológica Colombiana. Bogotá, Colombia.

${ }^{(2)}$ Residente de Primer año de Medicina Interna, Pontificia Universidad Javeriana, Hospital Universitario San Ignacio, Bogotá, Colombia. Correspondencia: Abraham Alí Munive, correo electrónico: aali@neumologica.org. Recibido: 06/09/14. Aceptado: 10/09/14

\section{Referencia}

Blum CA, Nigro N, Briel M, Schuetz P, Ullmer E, Suter-Widmer I, et al. Adjunct prednisone therapy for patients with community-acquired pneumonia: a multicentre, double blind, randomised, placebo-controlled trial. Lancet. 2015; 385:1511-8.

\section{Pregunta}

¿Cuál es el beneficio de adicionar corticoides sistémicos comparado con placebo para el tratamiento de pacientes con neumonía adquirida en la comunidad? ¿Reduce el tiempo hacia la estabilidad clínica?

\section{Diseño}

Ensayo clínico aleatorizado, doble ciego, multicéntrico, controlado con placebo.

\section{Cegamiento}

Pacientes y clínicos evaluadores.

Periodo de seguimiento

53 meses.
Lugar

7 hospitales de tercer nivel en Suiza.

\section{Pacientes}

785 pacientes (edad promedio de 74 años, 487 (62\%) hombres) con diagnóstico de neumonía adquirida en la comunidad definida por nuevo infiltrado en la radiografía de tórax y la presencia de alguno de los siguientes signos y síntomas: tos, producción de esputo, disnea, temperatura corporal $>38^{\circ} \mathrm{C}$, auscultación pulmonar anormal o estertores, conteo leucocitario $>10.000$ cels o $<4.000$ cels.

\section{Intervención}

Los pacientes fueron asignados de manera aleatoria (razón 1:1) para recibir prednisona $50 \mathrm{mg}$ día durante siete días o placebo. Prednisona $(n=392)$ o placebo $(n=393)$.

\section{Resultados}

Tras la realización del análisis por intención de tratar se evidenció 
que el tiempo promedio hacia la estabilidad clínica (días hasta la normalización de los signos vitales durante 24 horas o más) fue significativamente más corto en el grupo de prednisona (3,0 días; IQR 2,5$3,4)$ que en el placebo $(4,4$ días; IQR 4,0-5,0), con un HR de 1,33 (95\% IC 1,15-1,50, p<0,0001).

El tiempo promedio para el egreso hospitalario fue más corto para el grupo de prednisona. La duración total del tratamiento antibiótico no mostró diferencias entre ambos grupos, pero la duración del antibiótico endovenoso fue menor en el grupo de prednisona. Los niveles séricos de proteína $\mathrm{C}$ reactiva fueron significativamente más bajos en el grupo de prednisona a los 3, 5 y 7 días, respectivamente (tabla 1 ).

La mortalidad a 30 días por todas las causas no difirió entre los grupos. La incidencia de cualquier evento adverso en relación con el uso de corticoide fue mayor en el grupo de prednisona, específicamente la frecuencia de hiperglucemia intrahospitalaria que requirió el uso de insulina.

\section{Conclusión}

El tratamiento con prednisona durante 7días en pacientes hospitalizados con neumonía adquirida en la comunidad, reduce el tiempo hacia la estabilidad clínica, la estancia hospitalaria y la duración del tratamiento endovenoso, con mayor frecuencia de hiperglucemia.

\section{Comentario}

El empleo de los corticoides sistémicos en neumonía adquirida de la comunidad teóricamente podría tener beneficio por su efecto antiinflamatorio; sin embargo, a pesar de la base racional, su uso es aún controvertido y existen pocos estudios clínicos aleatorizados controlados con placebo que evalúen los beneficios de esta terapia de forma adjunta o complementaria al tratamiento antibiótico convencional (1).

Hasta el momento se han llevado a cabo dos revisiones sistemáticas y tres metaanálisis en los cuales se concluye que la terapia con corticoides en neumonía adquirida en la comunidad puede ofrecer beneficios; no obstante, no existe hasta el momento un estudio aleatorizado con suficiente poder que lo compruebe (2-4).

Fue por este motivo que Blum y colaboradores decidieron investigar a través de este estudio de efectos a corto plazo de la prednisona comparándola con placebo en pacientes hospitalizados por neumonía adquirida en la comunidad, queriendo evaluar el tiempo que se tardaría en lograr la estabilidad clínica como desenlace primario.

El estudio mostró que el uso de prednisona 50 $\mathrm{mg}$ /día durante 7 días en pacientes con neumonía adquirida en la comunidad redujo 1,4 días el tiempo en lograr la estabilidad clínica así como el tiempo de estancia hospitalaria en 1 día y la duración del tratamiento con antibiótico endovenoso. Adicionalmente, la terapia con prednisona fue bien tolerada, mostrando únicamente un aumento transitorio en la tasa de hiperglucemia intrahospitalaria y la necesidad de uso de insulina para corregirlo, sin incrementar el tiempo de estancia total.

En un sistema de salud como el colombiano, obtener un día menos de hospitalización y retiro

Tabla 1. Desenlaces de los grupos de prednisona y placebo.

\begin{tabular}{|lcrrr|}
\hline \multicolumn{1}{|c}{ Desenlaces } & $\begin{array}{c}\text { Prednisona } \\
(\mathbf{n = 3 9 2 )}\end{array}$ & $\begin{array}{c}\text { Placebo } \\
(\mathbf{n = 3 9 3 )}\end{array}$ & Hazard ratio & Valor $\mathbf{p}$ \\
Tiempo hacia la estabilidad clínica, días & $3,0(2,5-3,4)$ & $4,4(4,4-5,0)$ & HR 1,33 (1,15 a 1,50) & $<0,0001$ \\
$\begin{array}{l}\text { Tiempo efectivo de egreso hospitalario, } \\
\text { días }\end{array}$ & $6,0(6,0-7,0)$ & $7,0(7,0-8,0)$ & HR 1,19 (1,04 a 1,38) & 0,012 \\
Tratamiento antibiótico IV, días & $4,0(3,0-6,0)$ & $5,0(3,0-7,0)$ & $\begin{array}{r}\text { Diferencia }-0,89 \text { días } \\
(-1,57 \text { a }-0,20 \text { días })\end{array}$ & 0,011 \\
Muerte por cualquier causa & $16(4 \%)$ & $13(3 \%)$ & OR 1,24 (0,59 a 2,62) & 0,57 \\
\hline
\end{tabular}


anticipado del antibiótico endovenoso, supone un aporte importante. La escasez de camas y recursos genera la posibilidad que este estudio tenga aplicabilidad en nuestro medio. Los efectos secundarios relacionados con la hiperglicemia obligan a que no se aplique la terapia en el grupo de pacientes con trastornos previos de la glicemia.

\section{Bibliografía}

1. Rodríguez de Castro y Solé-Violán. Corticoides en la neumonía adquirida en la comunidad. Argumentos en contra. Arch Bronconeumol. 2011; 47(5): 219-21.

2. Lamontagne F, Briel M, Guyatt GH, Cook DJ, Bhatnagar N, Meade M. Corticosteroid therapy for acute lung injury, acute respiratory distress syndrome, and severe pneumonia: a meta-analysis of randomized controlled trials. J Crit Care. 2010;25:420-35.

3. Nie W, Zhang Y, Cheng J, Xiu Q. Corticosteroids in the treatment of community-acquired pneumonia in adults: a meta-analysis. Plos One. 2012; 7:e47926.

4. Shafiq M, Mansoor MS, Khan AA, Sohail MR, Murad MH. Adjuvant steroid therapy in community-acquired pneumonia: a systematic review and meta-analysis. J Hosp Med. 2013;8:68-75. 